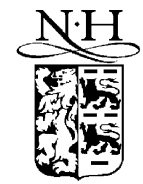

ELSEVIER
Available at

www.ComputerScienceWeb.com

powered by SCience @direct.
FGCS

Puture

GENERATION

SYSTEMS

Future Generation Computer Systems 19 (2003) 1221-1230

\title{
A numerically reliable approach to robust pole assignment for descriptor systems
}

\author{
A. Varga \\ German Aerospace Center, DLR_Oberpfaffenhofen, Institute of Robotics and Mechatronics, D-82234 Wessling, Germany
}

\begin{abstract}
We propose a general, numerically reliable computational approach to solve the pole and eigenstructure assignment problem for descriptor systems. In the multi-input case, the proposed approach addresses the intrinsic non-uniqueness of the pole assignment problem solution by simultaneously minimizing the sensitivity of the feedback gain and of closed-loop eigenvalues. For this purpose, a minimum norm robust pole assignment problem is formulated and solved as an unconstrained minimization problem for a suitably chosen cost function. By using a generalized Sylvester equation-based parameterization, an explicit expression of the gradient of the cost function is derived to allow the efficient solution of the minimization problem by using powerful gradient search-based minimization techniques.
\end{abstract}

(C) 2003 Published by Elsevier Science B.V.

Keywords: Descriptor systems; Pole assignment; Numerical methods

\section{Introduction}

Pole assignment techniques to modify the dynamic response of linear systems are among the most studied problems in modern control theory. The complete theoretical solution of this problem for standard systems has been followed by the development of many computational methods (see for example, the collection of reprints in [13]). Sensitivity analysis of the pole assignment problem (see [10] and references therein) moves one step forward the understanding of difficulties and practical limitations associated with the usage of solution methods.

We address the solution of the pole assignment problem for the following descriptor system:

$E \lambda x(t)=A x(t)+B u(t)$,

where $x(t) \in \mathbb{R}^{n}, u(t) \in \mathbb{R}^{m}, A, B, E$ are real matrices of appropriate dimensions with $E$ square and

E-mail address: andras.varga@dlr.de (A. Varga). generally singular, $\lambda x(t)=\dot{x}(t)$ for a continuous-time system, and $\lambda x(t)=x(t+1)$ for a discrete-time system. We assume that $r=\operatorname{rank} E$, the pencil $A-\lambda E$ is regular, i.e. $\operatorname{det}(A-\lambda E) \not \equiv 0$, and the system (1) is controllable, i.e. $\operatorname{rank}(A-\lambda E, B)=n$ for $\lambda \in \mathbb{C}$ and $\operatorname{rank}\left[\begin{array}{ll}E & B\end{array}\right]=n$. In what follows we denote with $\Lambda(A, E)$ the set of generalized eigenvalues of the pair $(A, E)$. Note that in general there exist at least $r$ infinite eigenvalues. If the number $n_{\infty}$ of infinite eigenvalues exceeds $r$, then $n_{\infty}-r$ eigenvalues are called the impulsive modes of the system.

We consider the following descriptor eigenvalue assignment problem (DEAP).

$D E A P$. Given a set of $n$ self-conjugate complex numbers $\Gamma_{n}=\left\{\lambda_{1}, \ldots, \lambda_{n}\right\}$ (some values could possibly be infinite), determine the gain matrices $F$ and $K$ in the proportional-derivative feedback

$u(t)=F x(t)-K \lambda x(t)+v(t)$

such that the closed-loop system pencil $A+B F-$ $\lambda(E+B K)$ is regular and 
$\Lambda(A+B F, E+B K)=\Gamma_{n}$.

One of the main applications of the DEAP is in solving several coprime factorizations problems of general rational matrices $[12,16]$. For example, by choosing $\Gamma_{n}$ with only finite elements, coprime factorizations with proper factors can be computed, while for $\Gamma_{n}$ with only infinite values, coprime factorizations with polynomial factors result.

A particular DEAP with constant-ratio proportionalderivative feedback

$u(t)=F(\cos \theta x(t)-\sin \theta \lambda x(t))+v(t)$

has been studied in [20]. The solution of the DEAP with this type of feedback has interesting applications in solving minimum-degree coprime factorizations problems [12]. The proportional state feedback

$u(t)=F x(t)+v(t)$

can be used to assign the maximum number $r$ of finite eigenvalues $[8,14]$, by moving all impulsive modes of the open-loop system to arbitrary finite positions. The solution of this DEAP represents the first step to compute normalized coprime factorizations of general rational matrices by the methods proposed in [17]. Finally, the standard eigenvalue assignment problem corresponds to $E=I, K=0$, and $\Gamma_{n}$ having only finite values.

A generalized Schur method to solve the DEAP has been proposed in [16]. This method is based on the generalized real Schur form (GRSF) of the pair $(A, E)$ which allows a sequential eigenvalue assignment, by combining low order eigenvalue assignment (of orders 1 or 2) with reordering of diagonal blocks of a pair in GRSF. For the solution of the constant-ratio proportional-derivative DEAP, a similar technique can be employed. Alternatively, with the help of a bilinear transformation (see [12,20]), the problem can be reduced to a problem with invertible $E$ and solved, using the generalized Schur method with proportional feedback [15].

None of the above algorithms is able to exploit the intrinsic non-uniqueness of the DEAP in the multi-input case by imposing additional conditions when solving this problem. One aspect which is desirable from a practical point of view is to determine feedback matrices with small gains. Intuitively, this must be advantageous since small feedback gains lead to smaller control signals, and thus to less energy consumption. Small gains are also beneficial to reduce noise amplification. A second aspect important in pole assignment is to achieve a small condition number for the eigenvector matrices of the closed-loop system pencil. This is the goal of robust pole assignment as formulated for standard systems [7] (see also [2] for a recent survey) and descriptor systems [8]. Both these aspects are decisive for the overall sensitivity of assigned eigenvalues, because, as was shown in the standard systems case [10], high feedback gains or high condition numbers lead to increased sensitivity of the closed-loop eigenvalues. It appears thus that the simultaneous minimization of the feedback norm and condition of eigenvector matrix is a desirable general goal for solving the DEAP.

The solution of robust DEAP has been addressed in [4] for the case of distinct finite eigenvalues in $\Gamma_{n}$. The solution approach relies on a parameterization involving a preliminary polynomial right coprime factorization of the rational matrix $(\lambda E-A)^{-1} B$. Using this factorization, an eigenvector matrix is constructed which depends explicitly on a set of free parameters. These parameters are then adjusted using minimization techniques to ensure low sensitivity of eigenvalues. The main limitations of this approach are its inability to address the case of infinite eigenvalues and of multiple real eigenvalues. Furthermore, the computation of the preliminary factorization is in general a difficult computational problem for high order systems.

The solutions of DEAP proposed in $[8,14]$ address the case of impulse-free pure proportional feedback. In essence, the algorithm of [14] relies on the techniques of [8], but the solution provided in [14] covers only the case of simple eigenvalues at infinity $\left(n_{\infty}=\right.$ $r$ ). The solution approach of [8] to the robust DEAP computes first a suitable eigenvector matrix for the closed-loop eigenvalues. This is done iteratively, by improving successively the condition numbers characterizing the sensitivity of closed-loop eigenvalues. After convergence, the feedback is determined by solving two linear equations. The difficulties of this approach lie mainly in the selection of the eigenvectors and in the possible slow convergence of the iterative selection schemes. Moreover, since the selection methods are primarily intended for distinct eigenvalues, they can hardly address the case of multiple eigenvalues. 
Thus, this approach cannot be considered a general method. All above-mentioned methods do not address the norm minimization aspect, which is also essential for achieving a low sensitivity of the eigenvalue assignment problem.

In this paper, we propose a general, numerically reliable approach to solve the DEAP in its most general setting. Our approach explicitly addresses the computation of minimum norm state feedback which solves the DEAP and simultaneously minimizes the sensitivity of the closed-loop eigenvalues. The solutions of the DEAP relies on a Sylvester system-based parameterization. By specifying a set of free parameters, the closed-loop eigenvector matrix results as the solution of a generalized Sylvester system. The free parameters can be adjusted by an optimization-based search to ensure low norm feedback gains and well-conditioned eigenvector matrix. For the efficient solution of the minimization problems, powerful unconstrained minimization methods based on gradient search techniques can be employed. For this purpose, we derived an explicit expression of the gradient of the cost function. We discuss several functional and numerical features of the proposed approach, as generality, flexibility (for example, to assign a desired eigenstructure or to perform a partial pole assignment), numerical stability, computational efficiency. Numerical examples illustrate some of the features of the proposed approach to solve DEAPs.

\section{Solution of DEAP}

Our approach is based on a straightforward Sylvester equation-based parametrization of the DEAP. Let $F$ and $K$ be matrices which solve the DEAP. Then, there must exist invertible matrices $X$ and $Y$ such that

$$
Y^{-1}(A+B F) X=\tilde{A}, \quad Y^{-1}(E+B K) X=\tilde{E},
$$

where the matrices $\tilde{A}$ and $\tilde{E}$ are such that $\Lambda(\tilde{A}, \tilde{E})=$ $\Gamma_{n}$. If we define $G:=F X$ and $L:=K X$, then (2) can be rewritten as the following Sylvester system of matrix equations to be satisfied by $X$ and $Y$ :

$A X-Y \tilde{A}+B G=0, \quad E X-Y \tilde{E}+B L=0$.

Now we can try to solve the DEAP assuming that $\tilde{A}$ and $\tilde{E}$ are chosen such that $\Lambda(\tilde{A}, \tilde{E})=\Gamma_{n}$, and $G$ and
$L$ are given parameter matrices. To solve the DEAP, we need to solve (3) for $X$ and $Y$ and, provided $X$ and $Y$ are invertible, we compute the feedback matrices as

$F=G X^{-1}, \quad K=L X^{-1}$.

To enforce the invertibility of $X$ and $Y$, we conjecture, on basis of results in [3], that the matrices $\tilde{A}, \tilde{E}, G$ and $L$ must fulfill the conditions: (1) the pair $(\tilde{A}-$ $\lambda \tilde{E}, G-\lambda L)$ is observable; $(2) \Lambda(A, E) \cap \Lambda(\tilde{A}, \tilde{E})=$ $\emptyset$. These conditions together with the controllability of pair $(A-\lambda E, B)$ ensure that $X$ and $Y$ satisfying (3) are generically invertible.

If the pair $(\tilde{A}, \tilde{E})$ is in a Weierstrass form, then $X$ and $Y$ play the roles of the eigenvector matrices for the closed-loop system pair $(A+B F, E+B K)$. In light of the sensitivity results in [10] for standard systems, it is meaningful to exploit the non-uniqueness of the DEAP for multi-input systems by minimizing additionally the sensitivity of the closed-loop eigenvalues and the norm of the feedback matrix. This leads to a minimum norm robust DEAP for which we propose a solution method combining unconstrained optimization techniques with the parametric Sylvester equation-based approach.

As a measure of the sensitivity of closed-loop eigenvalues, we use the condition numbers $\kappa_{F}(X)$ and $\kappa_{F}(Y)$ of $X$ and $Y$ with respect to the Frobenius norm. For computational convenience, instead of minimizing $\kappa_{F}(X):=\|X\|_{F}\left\|X^{-1}\right\|_{F}$, the minimization of the sum $\|X\|_{F}^{2}+\left\|X^{-1}\right\|_{F}^{2}$ can be alternatively performed, since the two optimization problems are mathematically equivalent [1]. Thus, for the simultaneous minimization of the norm of the state feedback matrices $F$ and $K$ and of the two condition numbers $\kappa_{F}(X)$ and $\kappa_{F}(Y)$ we can use the following performance index:

$$
\begin{aligned}
J= & \frac{1}{2} \alpha\left(\|X\|_{F}^{2}+\left\|X^{-1}\right\|_{F}^{2}+\|Y\|_{F}^{2}+\left\|Y^{-1}\right\|_{F}^{2}\right) \\
& +\frac{1}{2}(1-\alpha)\left(\|F\|_{F}^{2}+\|K\|_{F}^{2}\right),
\end{aligned}
$$

where $0 \leq \alpha \leq 1$ is a weighting factor. For $\alpha=0$, $J$ defines a pure norm minimization problem, while for $\alpha=1$ we get a pure robust DEAP. Intermediary values of $\alpha$ lead to a combination of both aspects.

The main advantage of the Sylvester equation-based parameterization is that it allows a straightforward derivation of analytic expressions of gradients of the performance criterion $J$ with respect to the free 
parameters $G$ and $L$. We have the following result for the DEAP (for the proof see Appendix A).

Proposition 1. Let $(F, K)$ be the pair of state feedback matrices computed as in (4), assigning the desired eigenvalues $\Gamma_{n}$ for given $(\tilde{A}, \tilde{E})$ and $(G, L)$. Then, the gradients of $J$ with respect to $G$ and $L$ are given by

$\nabla_{G} J=(1-\alpha) F X^{-\mathrm{T}}+B^{\mathrm{T}} U$,

$\nabla_{L} J=(1-\alpha) K X^{-\mathrm{T}}+B^{\mathrm{T}} V$,

where $U$ and $V$ satisfy the Sylvester system:

$A^{\mathrm{T}} U+E^{\mathrm{T}} V=S, \quad U \tilde{A}^{\mathrm{T}}+V \tilde{E}^{\mathrm{T}}=-T$

for

$$
\begin{aligned}
S= & (1-\alpha)\left(F^{\mathrm{T}} F+K^{\mathrm{T}} K\right) X^{-\mathrm{T}} \\
& +\alpha\left(-X+X^{-\mathrm{T}} X^{-1} X^{-\mathrm{T}}\right), \\
T= & \alpha\left(-Y+Y^{-\mathrm{T}} Y^{-1} Y^{-\mathrm{T}}\right) .
\end{aligned}
$$

Each function and gradient evaluation involves the solution of the two Sylvester systems (3) and (7). An efficient algorithm to solve these equations is available [6]. In the next section, we describe a transformation-based approach by which gradient computations can be substantially speeded up.

Having explicit analytical expressions for the function and its gradient it is easy to employ any gradient-based technique to minimize $J$. However, since the dimension of the minimization problem, $2 \mathrm{~nm}$, could be large, a particularly well-suited class of methods to use is the class of unconstrained descent methods, as for instance, the limited memory BFGS method [9] used in conjunction with a line search procedure with guaranteed decrease [11]. The guaranteed decrease feature of these methods ensures that for $\alpha>0$ the condition numbers $\kappa_{F}(X)$ and $\kappa_{F}(Y)$ progressively decrease and thus the solutions $X$ and $Y$ of (3) remain invertible at each iteration once invertible solutions have been determined at the first iteration.

Remark 2. When using gradient techniques to solve DEAPs by function minimization, it is likely that the computed solution is only a local minimum. One way to try to cure this aspect is by solving the problem repeatedly with different initializations, and choosing that solution which produces the lowest value of the cost function. Note however, that in most of cases the global minimum leads to condition numbers of the transformation matrices $X$ and $Y$ which have the same order of magnitude as those corresponding to any of local minima. Thus there is practically no difference for solving a robust eigenvalue assignment problem if the global minimum or one of local minima is employed to compute the feedback.

\section{Algorithmic features}

A satisfactory eigenvalue assignment algorithm must fulfill several requirements to serve as basis for a numerically robust software implementation. In what follows we discuss the main algorithmic features of the Sylvester equation approach, as generality, flexibility, numerical stability, computational efficiency.

\subsection{Generality}

A general eigenvalue assignment algorithm must be able to assign an arbitrary set of eigenvalues. Furthermore, in an ideal case, such an algorithm would also be able to assign a desired eigenstructure for the closed-loop system. Although the first requirement seems to be trivial, even well-known methods implemented in commercial software do not fulfill this requirement. For example, the robust pole assignment methods of [8] cannot assign poles with multiplicities greater than the rank of $B$. In what follows, we show that the Sylvester approach allows both the assignment of an arbitrary set of eigenvalues and of a given admissible eigenstructure for the closed-loop eigenvalues.

An arbitrary set of eigenvalues $\Gamma_{n}$ can be assigned with the Sylvester equation-based approach by suitably choosing the matrix pair $(\tilde{A}, \tilde{E})$. Suppose $\Gamma_{n}$ contains $p$ distinct eigenvalues $\lambda_{1}, \ldots, \lambda_{p}$, and each eigenvalue $\lambda_{i}$ has multiplicity $k_{i}$. If infinite eigenvalues are present, assume that $\lambda_{p}=\infty$. We can choose the pair $(\tilde{A}, \tilde{E})$ in a Weierstrass canonical form:

$\tilde{A}-\lambda \tilde{E}$

$$
=\left[\begin{array}{cccc}
J_{k_{1}}\left(\lambda_{1}\right)-\lambda I & 0 & 0 \\
\vdots & \ddots & \vdots & \vdots \\
0 & & J_{k_{p-1}}\left(\lambda_{p-1}\right)-\lambda I & 0 \\
0 & & 0 & I-\lambda J_{k_{p}}(0)
\end{array}\right],
$$


where $J_{k}(\lambda)$ denotes a Jordan block of order $k$ for the eigenvalue $\lambda$. For a complex eigenvalue $\lambda_{i}$ belonging to a multiple pair of complex conjugated eigenvalues $\left(\lambda_{i}, \bar{\lambda}_{i}\right)$, a $2 k_{i} \times 2 k_{i}$ real Jordan block can be used instead of two $k_{i} \times k_{i}$ complex Jordan blocks [5, p. 365]. If rank $B=m>1$, better conditioned transformation matrices $X$ and $Y$ can be obtained by employing several Jordan blocks of lower dimensions for each multiple eigenvalue. Thus for each $\lambda_{i}$ of multiplicity $k_{i}$, up to $m$ Jordan blocks with dimensions at most $\left[k_{i} / m\right]+1$ can be used, where [.] denotes the integer part.

If the desired closed-loop spectrum $\Gamma_{n}=\Lambda(\tilde{A}, \tilde{E})$ is disjoint from the open-loop spectrum $\Lambda(A, E)$, then by choosing suitable $G$ and $L$, the Sylvester system (3) has the unique solution $X$ and $Y$ with $X$ invertible, and the solution matrices $F$ and $K$ are computed from (4). The case of overlapping open-loop and closed-loop spectra can be easily addressed by means of a preliminary state-feedback with $F_{0}$ and $K_{0}$ chosen such that $\Gamma_{n}$ and $\Lambda\left(A+B F_{0}, E+B K_{0}\right)$ are disjoint (as a practical solution, two randomly generated matrices $F_{0}$ and $K_{0}$ can be used). If we solve the DEAP for the modified pair $\left(A+B F_{0}-\lambda\left(E+B K_{0}\right), B\right)$ and $\left(F_{1}, K_{1}\right)$ is the corresponding solution, then $(F, K)=\left(F_{0}+\right.$ $\left.F_{1}, K_{0}+K_{1}\right)$ is the solution of the original problem.

\subsection{Flexibility}

The Sylvester equation approach in conjunction with the optimization-based search for a minimum norm and well-conditioned feedback exploits the intrinsic freedom of the multi-input DEAP to address additional requirements, as for example, the conditioning aspect of the eigenvalue assignment problem. Note that most of existing eigenvalue assignment algorithms do not have the flexibility to exploit this structural feature of the problem, and even algorithms for robust pole assignment, address only partially this aspect by ignoring completely the feedback norm minimization. Moreover, these methods have also restrictions with respect to the allocation of the closed-loop eigenstructure.

Another desirable feature of a flexible eigenvalue assignment method is to allow a partial eigenvalue assignment, i.e. to keep unmodified some of the open-loop eigenvalues while moving the rest of eigenvalues to desirable locations. Since the partial eigenvalue assignment is a very useful feature, especially in the case of stabilizing high order systems, we show how this feature can be easily accommodated within the Sylvester equation-based approach.

It is easy to see that the performance index $J$ is invariant to an orthogonal system similarity transformation, i.e. if $F$ and $K$ are the optimal feedback matrices for the descriptor pair $(A-\lambda E, B)$, then $\hat{F}=F Z$ and $\hat{K}=K Z$ are the optimal feedback matrices for the transformed pair $(\hat{A}-\lambda \hat{E}, \hat{B}):=$ $\left(Q^{\mathrm{T}} A Z-\lambda Q^{\mathrm{T}} E Z, Q^{\mathrm{T}} B\right)$, where $Q$ and $Z$ are orthogonal matrices. Thus, if we want to keep unmodified the generalized eigenvalues of the pair $(A, E)$ lying in a "good" region $\mathbb{C}_{\mathrm{g}}$ of $\mathbb{C}$ and to modify only those lying in its complement $\mathbb{C}_{\mathrm{b}}:=\mathbb{C} \backslash \mathbb{C}_{\mathrm{g}}$ (the "bad" region), then we can first reduce $(A, E)$ to an ordered GRSF to obtain the triple:

$$
\begin{aligned}
& Q^{\mathrm{T}} E Z=\left[\begin{array}{cc}
E_{11} & E_{12} \\
0 & E_{22}
\end{array}\right], \quad Q^{\mathrm{T}} A Z=\left[\begin{array}{cc}
A_{11} & A_{12} \\
0 & A_{22}
\end{array}\right], \\
& Q^{\mathrm{T}} B=\left[\begin{array}{c}
B_{1} \\
B_{2}
\end{array}\right],
\end{aligned}
$$

where $\Lambda\left(A_{11}, E_{11}\right) \subset \mathbb{C}_{\mathrm{g}}$ and $\Lambda\left(A_{22}, E_{22}\right) \subset \mathbb{C}_{\mathrm{b}}$. With this separation, we can perform a partial eigenvalue assignment by solving for the optimal solution $F_{2}$ and $K_{2}$ the DEAP for the reduced descriptor pair $\left(A_{22}-\lambda E_{22}, B_{2}\right)$. The overall optimal feedback matrices result as $F=\left[\begin{array}{ll}0 & F_{2}\end{array}\right] Z^{\mathrm{T}}$ and $K=\left[\begin{array}{ll}0 & K_{2}\end{array}\right] Z^{\mathrm{T}}$. This approach to the solution of the partial eigenvalue assignment problem represents a numerically sound approach to address DEAPs with overlapping openand closed-loop spectra.

\subsection{Numerical stability and accuracy}

The computation of the optimal solution $F$ and $K$ for the computed optimal parameter matrices $G$ and $L$ involves the solution of two systems of linear equations: the Sylvester system (3) to compute $X$ and $Y$ [6], and the linear system

$\left[\begin{array}{l}F \\ K\end{array}\right] X=\left[\begin{array}{l}G \\ L\end{array}\right]$

to compute the feedback matrices $F$ and $K$. Thus the Sylvester equation-based approach can be considered to be practically numerically stable. 
Concerning the accuracy of the results, in a robust pole assignment problem it is expected that for a carefully chosen set of closed-loop eigenvalues and corresponding eigenstructure the optimal $X$ is reasonably well-conditioned. Thus the last computational step to determine the feedback matrices in (4) is guaranteed to be very accurate. The main source of errors is the solution of the Sylvester system, and thus the separation of spectra of the pairs $(A, E)$ and $(\tilde{A}, \tilde{E})$ is the essential factor here. However, a good separation can always be achieved by an initial eigenvalue shifting with a preliminary feedback (see also Section 3.1) and a partial eigenvalue assignment can be performed in the case of overlapping spectra. Thus, for most practical problems we can expect that the computed results corresponding to an optimal solution are very accurate.

\subsection{Computational efficiency}

The overall efficiency of the algorithms heavily depends on the costs of function and gradient evaluations. Each function and gradient evaluation involves the solution of the two generalized Sylvester equations (3) and (7) sharing the same coefficient matrices. The standard procedure to solve these equations is the well-known generalized Schur method [6]. This approach can be efficiently employed in our case provided the pair $(A, E)$ is reduced first to a GRSF using an orthogonal similarity transformation and assuming further that the pair $(\tilde{A}, \tilde{E})$ is in a Weierstrass form (a particular GRSF). The reduction of $(A, E)$, performed only once, requires about $25 n^{3}$ operations and can be seamlessly combined with the reordering of the GRSF to accommodate with the partial eigenvalue assignment requirement. The solution of the minimization problem can be performed to obtain the optimal solutions $\hat{F}$ and $\hat{K}$ for the transformed descriptor pair $(\hat{A}-\lambda \hat{E}, \hat{B})=\left(Q^{\mathrm{T}} A Z-\lambda Q^{\mathrm{T}} E Z, Q^{\mathrm{T}} B\right)$ with the pair $(\hat{A}, \hat{E})$ in GRSF and $(\tilde{A}, \tilde{E})$ in Weierstrass form. The solution of the original DEAP results as $F=\hat{F} Z^{\mathrm{T}}$ and $K=\hat{K} Z^{\mathrm{T}}$. For the transformed problem, the function and gradient evaluations can be performed very efficiently since now we have to solve only reduced Sylvester equations with the coefficient matrices in GRSF. This involves about $2 n^{3}$ operations for the solution of each Sylvester system by using the algorithm of [6]. Thus the overall cost to evaluate the function and its gradient is about $10 n^{3}$ operations, from which
$6 n^{3}$ operations account to form the free terms $S$ and $T$ in (7).

\section{Numerical examples}

Consider the system from [8] with the matrices

$$
\begin{aligned}
A & =\left[\begin{array}{ccccc}
0 & 1.1 & 0 & 0 & 0 \\
0 & 0 & 1.56 & 0 & 0 \\
1.23 & 0 & 0 & 1.98 & 0 \\
0 & 0 & 0 & 0 & 0 \\
0 & 0 & 1.01 & 0 & 0
\end{array}\right], \\
E & =\left[\begin{array}{ccccc}
0 & 0 & 0 & 1.72 & 0 \\
0 & 0 & 0 & 0 & 0 \\
-0.82 & 0 & 0 & 0 & 0 \\
0 & 0 & 0 & 0 & 0 \\
0 & 0 & 0 & 0 & 1
\end{array}\right], \\
B & =\left[\begin{array}{cccc}
1.55 & 0 & 0 & 0 \\
0 & 1.07 & 0 \\
0 & 0 & -1.11 \\
0 & -2.5 & 0
\end{array}\right] .
\end{aligned}
$$

This system is not regular since $\operatorname{det}(A-\lambda E) \equiv$ 0 , but it can be made regular with a preliminary state feedback. We assigned the closed-loop eigenvalue set $\Gamma_{5}=\{-0.5,-1,-2, \infty, \infty\}$ using both proportional-derivative feedback as well as pure proportional feedback. This example illustrates not only the ability of the new approach to compute well-conditioned (sometimes almost orthogonal) eigenvector matrices, but also its ability to solve the DEAP even for non-regular systems or to solve the DEAP in the case when infinite eigenvalues are present in both the open-loop and closed-loop systems.

For the solution of DEAP, we chose $\tilde{A}=$ $\operatorname{diag}(-0.5,-1,-2,1,1), \tilde{E}=\operatorname{diag}(1,1,1,0,0)$ and solved the minimization problem for $J$ for several values of $\alpha$. For the resulting optimal solutions, we computed in each case the 2-norm condition numbers $\kappa_{2}(X)$ and $\kappa_{2}(Y)$, and the 2-norm of the compound feedback matrix [ $\left.\begin{array}{ll}F & K\end{array}\right]$. The results are given in Table 1. It is easy to observe that for decreasing values of $\alpha$ the norms of feedback matrices decrease, but the 
Table 1

Results for the proportional-derivative feedback DEAP

\begin{tabular}{|c|c|c|c|}
\hline$\alpha$ & $\left\|\left[\begin{array}{ll}F & K\end{array}\right]\right\|_{2}$ & $\kappa_{2}(X)$ & $\kappa_{2}(Y)$ \\
\hline 1 & 1.35 & 3.75 & 1.57 \\
\hline 0.1 & 0.58 & 3.97 & 3.18 \\
\hline 0.01 & 0.33 & 4.25 & 6.52 \\
\hline 0.001 & 0.21 & 5.06 & 16.98 \\
\hline 0.0001 & 0.13 & 9.86 & 57.07 \\
\hline 0 & 0.014 & 445 & $1.88 \times 10^{7}$ \\
\hline
\end{tabular}

condition numbers of eigenvector matrices increase. For pure norm minimization, a very small norm has been achieved but with a very ill-conditioned transformation matrix $Y$. Each solution for $\alpha$ in the range $\left[10^{-2}, 1\right]$ is practically acceptable.

For reference purpose, we give the feedback matrices computed for the pure robust $\operatorname{DEAP}(\alpha=1)$ :

$$
\begin{aligned}
F & =\left[\begin{array}{ccccc}
0.0713 & -0.2135 & -0.1447 & 0.6371 & -0.1770 \\
-0.0708 & 0.1770 & 0.4130 & 0.3929 & 0.0373 \\
-0.2483 & -0.2858 & 0.0012 & -0.3999 & 0.9293
\end{array}\right], \\
K & =\left[\begin{array}{ccccc}
0.0705 & 0.1758 & -0.5965 & -0.2388 & -0.0443 \\
0.1425 & 0.0033 & -0.0111 & -0.4988 & 0.3255 \\
0.3163 & 0.0623 & -0.2114 & 0.4182 & -0.1678
\end{array}\right]
\end{aligned}
$$

The robustness of the solution can be easily checked by computing the eigenvalues of the pair $(A+B F, E+$ $B K$ ) for the matrices $F$ and $K$ truncated to the displayed four digits. The resulting closed-loop eigenvalues are

$$
\{-1.999997,-0.999998,-0.50004, \infty, \infty\}
$$

and thus are accurate to five decimal digits.

To compare our approach with the method of [8], we solved the DEAP by using only proportional feedback. The results are given in Table 2. The same tendencies for decreasing norms of $F$ and increas-

Table 2

Results for proportional feedback DEAP

\begin{tabular}{llrc}
\hline$\alpha$ & $\|F\|_{2}$ & $\kappa_{2}(X)$ & $\kappa_{2}(Y)$ \\
\hline 1 & 1.79 & 4.23 & 2.88 \\
0.1 & 0.81 & 4.52 & 4.88 \\
0.01 & 0.47 & 5.18 & 9.61 \\
0.001 & 0.28 & 9.47 & 27.01 \\
0.0001 & 0.17 & 19.86 & 96.39 \\
0 & 0.0096 & 376.6 & $5.48 \times 10^{7}$ \\
\hline
\end{tabular}

ing ill-conditioning with decreasing $\alpha$ values can be observed as in Table 1. Because of increased parametric freedom, the proportional-derivative feedback achieves smaller norms for the same ranges of the condition numbers.

For reference purpose, we give the proportional feedback matrix computed for the pure robust DEAP $(\alpha=1)$ :

$F=\left[\begin{array}{ccccc}-0.0584 & 0.2600 & -0.3888 & 0.6921 & 0.0467 \\ 0.0647 & 0.0406 & 0.3480 & 0.0364 & 0.4027 \\ 0.3249 & 1.0523 & -0.3263 & 1.1542 & -0.0544\end{array}\right]$.

The robustness of the solution can be easily checked by computing the eigenvalues of the pair $(A+B F, E)$ with the elements of matrix $F$ truncated to the displayed four digits. The resulting closed-loop eigenvalues are $\{-1.99985,-0.999997,-0.500033, \infty, \infty\}$

and thus are accurate to four decimal digits. This result is marginally better than that reported in [8] both with respect to the condition number of $X$ as well as the magnitude of $F$. Note, however, that practically the same robustness can be achieved for $\alpha=0.01$ with a four times smaller magnitude of the feedback matrix $F$.

\section{Conclusions}

We focused on developing a reliable numerical approach to exploit the intrinsic non-uniqueness of the DEAP. One possibility to address the non-uniqueness is by formulating the DEAP as a minimum norm robust pole assignment problem. By using a convenient parametrization, a solution of the DEAP is sought by minimizing a special cost function expressing the weighted requirements for minimum norm of the feedback matrix and the minimum sensitivity of the closed-loop eigenvalues. The derived explicit expression for the gradient of the cost function allows the use of standard gradient search-based minimization techniques. Similar expressions for the constant-ratio proportional-derivative feedback DEAP and pure proportional feedback DEAP have been derived in [19].

The efficient evaluation of the cost functions and gradients is of paramount importance for the usefulness of the proposed approach. Using transformation 
techniques in conjunction with the solution of reduced generalized Sylvester equations is the main ingredient to achieve this goal. Furthermore, it allows to address, with practically no extra costs, the partial pole assignment problem too. We believe that the proposed robust pole assignment approach is a viable way to solve large DEAPs in the perspective of the requirements formulated by recent sensitivity analysis results [10].

In a broader context, the Sylvester equation-based approach provides a unified framework to solve various eigenvalue assignment problems for standard, descriptor and even periodic systems [18]. In light of discussions on generality and flexibility, this approach has the potential to become the standard way to solve all classes of eigenvalue assignment problems.

\section{Appendix A. Proofs}

To prove Proposition 1 we need the following result.

Lemma A.1. Let $A, E \in \mathbb{R}^{n \times n}, \tilde{A}, \tilde{E} \in \mathbb{R}^{r \times r}$ and $C, D \in \mathbb{R}^{n \times r}$ be given such that $\Lambda(A, E) \cap \Lambda(\tilde{A}, \tilde{E})=$ $\emptyset$. Let $X \in \mathbb{R}^{n \times r}$ and $Y \in \mathbb{R}^{n \times r}$ be the solution of the Sylvester system:

$A X-Y \tilde{A}=C, \quad E X-Y \tilde{E}=D$.

Then for $S, T \in \mathbb{R}^{n \times r}$ we have $\operatorname{tr}\left[S^{\mathrm{T}} X+T^{\mathrm{T}} Y\right]=$ $\operatorname{tr}\left[U^{\mathrm{T}} C+V^{\mathrm{T}} D\right]$, where $U, V \in \mathbb{R}^{n \times r}$ satisfy the Sylvester system:

$A^{\mathrm{T}} U+E^{\mathrm{T}} V=S, \quad U \tilde{A}^{\mathrm{T}}+V \tilde{E}^{\mathrm{T}}=-T$.

Proof. By using Kronecker products and the notation $\bar{x}=\operatorname{vec}(X)$ for the $\operatorname{vec}(\cdot)$ operator, we can express the solution of (A.1) as

$\left[\begin{array}{l}\bar{x} \\ \bar{y}\end{array}\right]=P^{-1}\left[\begin{array}{l}\bar{c} \\ \bar{d}\end{array}\right]$,

where

$$
P=\left[\begin{array}{cc}
I_{r} \otimes A & -\tilde{A}^{\mathrm{T}} \otimes I_{n} \\
I_{r} \otimes E & -\tilde{E}^{\mathrm{T}} \otimes I_{n}
\end{array}\right] .
$$

We obtain successively

$$
\begin{aligned}
\operatorname{tr} & {\left[S^{\mathrm{T}} X+T^{\mathrm{T}} Y\right] } \\
& =\left[\begin{array}{ll}
\bar{s}^{\mathrm{T}} & \bar{t}^{\mathrm{T}}
\end{array}\right]\left[\begin{array}{l}
\bar{x} \\
\bar{y}
\end{array}\right]=\left[\begin{array}{ll}
\bar{q}^{\mathrm{T}} & \bar{r}^{\mathrm{T}}
\end{array}\right] P^{-1}\left[\begin{array}{c}
\bar{c} \\
\bar{d}
\end{array}\right] \\
& =\left[\begin{array}{l}
\bar{u}^{\mathrm{T}} \bar{v}^{\mathrm{T}}
\end{array}\right]\left[\begin{array}{l}
\bar{c} \\
\bar{d}
\end{array}\right],
\end{aligned}
$$

where

$P^{\mathrm{T}}\left[\begin{array}{c}\bar{u} \\ \bar{v}\end{array}\right]=\left[\begin{array}{l}\bar{q} \\ \bar{r}\end{array}\right]$.

However,

$P^{\mathrm{T}}=\left[\begin{array}{cc}I_{r} \otimes A^{\mathrm{T}} & I_{r} \otimes E^{\mathrm{T}} \\ -\tilde{A} \otimes I_{n} & -\tilde{E} \otimes I_{n}\end{array}\right]$

and thus (A.3) corresponds to the Sylvester system (A.2). We further have

$$
\begin{aligned}
& \operatorname{tr}\left[S^{\mathrm{T}} X+T^{\mathrm{T}} Y\right]=\left[\begin{array}{ll}
\bar{u}^{\mathrm{T}} & \bar{v}^{\mathrm{T}}
\end{array}\right]\left[\begin{array}{l}
\bar{c} \\
\bar{d}
\end{array}\right] \\
& =\operatorname{tr}\left[U^{\mathrm{T}} C+V^{\mathrm{T}} D\right] \text {. }
\end{aligned}
$$

Proof of Proposition 1. We write the cost function (5) in the form:

$J=(1-\alpha)\left(J_{1}+J_{2}\right)+\alpha\left(J_{3}+J_{4}+J_{5}+J_{6}\right)$,

where $J_{1}=(1 / 2) \operatorname{tr}\left[F^{\mathrm{T}} F\right], J_{2}=(1 / 2) \operatorname{tr}\left[K^{\mathrm{T}} K\right]$, $J_{3}=(1 / 2) \operatorname{tr}\left[X^{\mathrm{T}} X\right], J_{4}=(1 / 2) \operatorname{tr}\left[X^{-\mathrm{T}} X^{-1}\right], J_{5}=$ $(1 / 2) \operatorname{tr}\left[Y^{\mathrm{T}} Y\right], J_{6}=(1 / 2) \operatorname{tr}\left[Y^{-\mathrm{T}} Y^{-1}\right]$. It follows that for $Z=G$ or $Z=L$, we have

$\nabla_{Z} J=(1-\alpha)\left(\nabla_{Z} J_{1}+\nabla_{Z} J_{2}\right)+\alpha \sum_{i=3}^{6} \nabla_{Z} J_{i}$

For $J_{1}=(1 / 2) \operatorname{tr}\left[F^{\mathrm{T}} F\right]$, we deduce the gradients $\nabla_{G} J_{1}$ and $\nabla_{L} J_{1}$ from the first order variation:

$\Delta J_{1}:=\operatorname{tr}\left[\left(\nabla_{G} J_{1}\right)^{\mathrm{T}} \Delta G+\left(\nabla_{L} J_{1}\right)^{\mathrm{T}} \Delta L\right]=\operatorname{tr}\left[F^{\mathrm{T}} \Delta F\right]$. 
From (4), we get $\Delta F=\Delta G X^{-1}-G X^{-1} \Delta X X^{-1}$ and we have successively

$$
\begin{aligned}
\Delta J_{1} & =\operatorname{tr}\left[F^{\mathrm{T}}(\Delta G-F \Delta X) X^{-1}\right] \\
& =\operatorname{tr}\left[X^{-1} F^{\mathrm{T}}(\Delta G-F \Delta X)\right] \\
& =\operatorname{tr}\left[X^{-1} F^{\mathrm{T}} \Delta G\right]-\operatorname{tr}\left[X^{-1} F^{\mathrm{T}} F \Delta X\right] .
\end{aligned}
$$

From (3), it follows that $\Delta X$ satisfies the Sylvester system:

$A \Delta X-\Delta Y \tilde{A}=-B \Delta G$,

$E \Delta X-\Delta Y \tilde{E}=-B \Delta L$.

By using Lemma A.1, we can write $\operatorname{tr}\left[X^{-1} F^{\mathrm{T}} F \Delta X\right]=$ $-\operatorname{tr}\left[U_{1}^{\mathrm{T}} B \Delta G+V_{1}^{\mathrm{T}} B \Delta L\right]$, where for $i=1, U_{1}$ and $V_{1}$ satisfy the Sylvester system:

$A^{\mathrm{T}} U_{i}+E^{\mathrm{T}} V_{i}=S_{i}, \quad U_{i} \tilde{A}^{\mathrm{T}}+V_{i} \tilde{E}^{\mathrm{T}}=-T_{i}$

with $S_{1}=F^{\mathrm{T}} F X^{-\mathrm{T}}$ and $T_{1}=0$. We further obtain

$\Delta J_{1}=\operatorname{tr}\left[\left(X^{-1} F^{\mathrm{T}}+U_{1}^{\mathrm{T}} B\right) \Delta G+V_{1}^{\mathrm{T}} B \Delta L\right]$

from which the gradients of $J_{1}$ result as

$\nabla_{G} J_{1}=F X^{-\mathrm{T}}+B^{\mathrm{T}} U_{1}, \quad \nabla_{L} J_{1}=B^{\mathrm{T}} V_{1}$.

In a completely similar way we obtain

$\nabla_{G} J_{2}=B^{\mathrm{T}} U_{2}, \quad \nabla_{L} J_{2}=K X^{-\mathrm{T}}+B^{\mathrm{T}} V_{2}$,

where for $i=2, U_{2}$ and $V_{2}$ satisfy (A.5) with $S_{2}=$ $K^{\mathrm{T}} K X^{-\mathrm{T}}$ and $T_{2}=0$. By using Lemma A.1 repeatedly, we determine the gradients of $J_{i}$, for $i=3, \ldots, 6$ in the form:

$\nabla_{G} J_{i}=B^{\mathrm{T}} U_{i}, \quad \nabla_{L} J_{i}=B^{\mathrm{T}} V_{i}$,

where $U_{i}$ and $V_{i}$ satisfy (A.5) for appropriate $S_{i}$ and $T_{i}$. For $J_{3}=(1 / 2) \operatorname{tr}\left[X^{\mathrm{T}} X\right]$, we have $\Delta J_{3}=\operatorname{tr}\left[X^{\mathrm{T}} \Delta X\right]$ and from Lemma A.1 we get $S_{3}=-X$ and $T_{3}=0$. For the term $J_{4}=(1 / 2) \operatorname{tr}\left[X^{-\mathrm{T}} X^{-1}\right]$, we have

$$
\begin{aligned}
\Delta J_{4} & =-\operatorname{tr}\left[X^{-\mathrm{T}} X^{-1} \Delta X X^{-1}\right] \\
& =-\operatorname{tr}\left[X^{-1} X^{-\mathrm{T}} X^{-1} \Delta X\right]
\end{aligned}
$$

and again from Lemma A.1 we obtain $S_{4}=$ $X^{-\mathrm{T}} X^{-1} X^{-\mathrm{T}}$ and $T_{4}=0$. In a similar way, we obtain for $J_{5}$ and $J_{6}: S_{5}=0, T_{5}=-Y, S_{6}=0$ and $T_{6}=Y^{-\mathrm{T}} Y^{-1} Y^{-\mathrm{T}}$. The linearity of Sylvester equation allows to combine the different right-hand side terms in two unique terms:

$$
\begin{aligned}
& S=(1-\alpha)\left(S_{1}+S_{2}\right)+\alpha\left(S_{3}+S_{4}+S_{5}+S_{6}\right), \\
& T=(1-\alpha)\left(T_{1}+T_{2}\right)+\alpha\left(T_{3}+T_{4}+T_{5}+T_{6}\right)
\end{aligned}
$$

and to write the corresponding solutions $U$ and $V$ in the same form:

$U=(1-\alpha)\left(U_{1}+U_{2}\right)+\alpha\left(U_{3}+U_{4}+U_{5}+U_{6}\right)$,

$V=(1-\alpha)\left(V_{1}+V_{2}\right)+\alpha\left(V_{3}+V_{4}+V_{5}+V_{6}\right)$.

The matrices $U$ and $V$ satisfy the Sylvester system (7) and the corresponding expression of gradients are those in (6).

\section{References}

[1] R. Byers, S.G. Nash, Approaches to robust pole assignment, Int. J. Contr. 49 (1989) 97-117.

[2] E. Chu, Optimization and pole assignment in control system design, Int. J. Appl. Math. Comput. Sci. 11 (2001) 10351053.

[3] E. De Souza, S.P. Bhattacharyya, Controllability, observability and the solution of $A X-X B=C$, Lin. Alg. Appl. 39 (1981) 167-188.

[4] G.-R. Duan, R.J. Patton, Robust pole assignment in descriptor systems via proportional plus partial derivative state feedback, Int. J. Contr. 72 (1999) 1193-1203.

[5] D.E. Goldberg, P. Lancaster, L. Rodman, Invariant Subspaces of Matrices with Applications, Wiley, New York, 1986.

[6] B. Kågström, L. Westin, Generalized Schur methods with condition estimators for solving the generalized Sylvester equation, IEEE Trans. Automat. Contr. 34 (1989) 745-751.

[7] J. Kautsky, N.K. Nichols, P. Van Dooren, Robust pole assignment in linear state feedback, Int. J. Contr. 41 (1985) 1129-1155.

[8] J. Kautsky, N.K. Nichols, E.-W. Chu, Robust pole assignment in singular control systems, Lin. Alg. Appl. 121 (1989) 9-37.

[9] D.C. Liu, J. Nocedal, On the limited memory BFGS method for large scale optimization, Math. Program. 45 (1989) 503528.

[10] V.L. Mehrmann, H. Xu, An analysis of the pole placement problem. II. The multi input case, Electr. Trans. Numer. Anal. (ETNA) 5 (1997) 77-97. http://etna.mcs.kent.edu/.

[11] J.J. Moré, D.J. Thuente, On line search algorithms with guaranteed sufficient decrease, ACM Trans. Math. Software 20 (1994) 286-307.

[12] C. Oară, A. Varga, Minimal degree coprime factorization of rational matrices, SIAM J. Matrix Anal. Appl. 21 (1999) 245-278.

[13] R.V. Patel, A.J. Laub, P. Van Dooren (Eds.), Numerical Linear Algebra Techniques for Systems and Control, IEEE Press, New York, 1994. 
[14] V.L. Syrmos, F.L. Lewis, Robust eigenvalue assignment for generalized systems, Automatica 28 (1992) 12231228.

[15] A. Varga, On stabilization of descriptor systems, Syst. Contr. Lett. 24 (1995) 133-138.

[16] A. Varga, Computation of coprime factorizations of rational matrices, Lin. Alg. Appl. 271 (1998) 83-115.

[17] A. Varga, Computation of normalized coprime factorizations of rational matrices, Syst. Contr. Lett. 33 (1998) 37-45.

[18] A. Varga, Robust pole assignment techniques via state feedback, in: Proceedings of the CDC'2000, Sydney, Australia, 2000, pp. 4655-4660.

[19] A. Varga, Robust pole assignment for descriptor systems, in: Proceedings of the MTNS'2000 Symposium, Perpignan, France, 2000.
[20] Z. Zhou, M.A. Shayman, T.-J. Tarn, Singular systems: a new approach in the time domain, IEEE Trans. Automat. Contr. 32 (1987) 42-50.

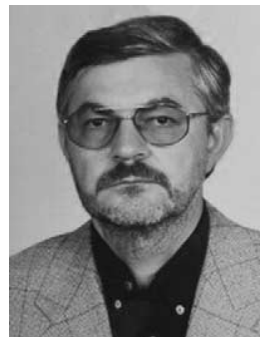

A. Varga has been with the German Aerospace Center (DLR) in Oberpfaffenhofen since 1993, where he is currently a Senior Scientist. His main research interests include the numerical methods for linear systems analysis and design, and robust numerical software for computer aided control system design. 